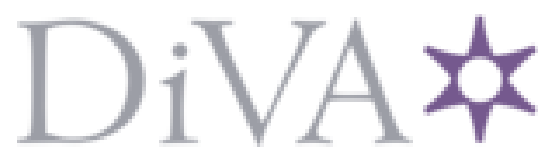

http://www.diva-portal.org

\title{
Postprint
}

This is the accepted version of a paper published in Biochemical and Biophysical Research Communications - BBRC. This paper has been peer-reviewed but does not include the final publisher proof-corrections or journal pagination.

Citation for the original published paper (version of record):

Hultqvist, G., Pedersen, S., Chi, C., Strømgaard, K., Gianni, S. et al. (2012)

An expanded view of the protein folding landscape of PDZ domains.

Biochemical and Biophysical Research Communications - BBRC, 421(3): 550-553

http://dx.doi.org/10.1016/j.bbrc.2012.04.042

Access to the published version may require subscription.

N.B. When citing this work, cite the original published paper.

Permanent link to this version:

http://urn.kb.se/resolve?urn=urn:nbn:se:uu:diva- 185575 


\section{An expanded view of the protein folding landscape of PDZ domains}

Greta Hultqvist ${ }^{1}$, Søren W. Pedersen ${ }^{1,2}$, Celestine Chi $^{1}$, Kristian Strømgaard ${ }^{2}$, Stefano Gianni $^{3}$ and Per Jemth ${ }^{1}$

${ }^{1}$ Department of Medical Biochemistry and Microbiology, Uppsala University, BMC Box 582, SE-75123 Uppsala, Sweden.

\footnotetext{
${ }^{2}$ Department of Drug Design and Pharmacology, University of Copenhagen, Universitetsparken 2, DK-2100 Copenhagen, Denmark
}

\footnotetext{
${ }^{3}$ Istituto Pasteur -Fondazione Cenci Bolognetti and Istituto di Biologia e Patologia Molecolari del CNR, Dipartimento di Scienze Biochimiche "A. Rossi Fanelli", Sapienza Università di Roma, Piazzale A. Moro 5, 00185 Rome, Italy
}

Corresponding author: Per Jemth

PerJemth@imbim.uu.se

Phone: +46-18-4714557

Fax: $+46-18-4714673$ 


\begin{abstract}
Most protein domains fold in an apparently co-operative and two-state manner with only the native and denatured states significantly populated at any experimental condition. However, the protein folding energy landscape is often rugged and different transition states may be rate limiting for the folding reaction under different conditions, as seen for the PDZ protein domain family. We have here analyzed the folding kinetics of two PDZ domains and found that a previously undetected third transition state is rate limiting under conditions that stabilize the native state relative to the denatured state. In light of these results, we have re-analyzed previous folding data on PDZ domains and present a unified folding mechanism with three distinct transition states separated by two high-energy intermediates. Our data show that sequence composition tunes the relative stabilities of folding transition states within the PDZ family, while the overall mechanism is determined by topology. This model captures the kinetic folding mechanism of all PDZ domains studied to date.
\end{abstract}

\title{
Keywords
}

PDZ domain, protein folding, folding kinetics, transition state, $\beta$-Tanford 


\section{Introduction}

Numerous protein-folding studies have demonstrated that protein domains have evolved to fold without accumulation of low-energy intermediates. Such low energy intermediates might aggregate through exposure of hydrophobic patches, which would explain why these intermediates are rare [1]. Nevertheless, the protein folding energy landscape is not a smooth surface, instead it is rugged and broad [2-4]. This ruggedness is reflected in the non-linear folding kinetics, where different transition states are rate limiting under different conditions, for example at low and high concentration of denaturant or in presence of stabilizing salts such as sodium sulfate. One type of protein domain, for which several members display such non-linear folding kinetics is the PDZ family of protein domains [5-7] (Fig. 1). PDZ domains are very common protein-protein interaction modules that usually bind the $\mathrm{C}$-terminus of target proteins $[8,9]$. We have previously studied the folding mechanisms of several members of this family [5] and found that they all folded in a reaction involving (at least) two transition states. These transition states had a similar compaction across the family, as reflected in their $\beta$-Tanford $\left(\beta_{\mathrm{T}}\right)$ values. However, one of the members of the family, neuronal nitric oxide synthase (nNOS) PDZ, fitted less well to the first of the two transition states, suggesting that the published model did not encompass its folding kinetics. Here, we have looked at the folding kinetics of two other members of the PDZ family, Postsynaptic density protein-95 (PSD-95) PDZ2 and Synapseassociated protein 97 (SAP97) PDZ2 in presence of sodium sulfate, that is, conditions that favor the native state relative to the denatured state. The results demonstrate that an early transition state becomes destabilized and thus rate limiting for folding under these conditions. Based on the new data we develop our previous model to entail three 
transition states, separated by two high-energy intermediates. Our new model reconciles the data for $\mathrm{nNOS}$ PDZ and is applicable to all studied members of the PDZ family.

\section{Materials and Methods}

\subsection{Protein expression and purification}

Expression and purification of the proteins PSD-95 PDZ2 with the mutation Y190W [5] and SAP97 PDZ2 with the mutations I342W and C378A [10] were performed as previously described.

\subsection{Kinetic folding experiments}

Stopped-flow experiments were performed on an apparatus from Applied Photophysics (SX-17 upgraded to SX-20) (Leatherhead, UK). Unfolding of the PDZ domains was achieved by rapid mixing of protein with buffer-urea solutions $(1: 10)$ in a stopped flow apparatus. Similarly, refolding was achieved by mixing a protein-urea solution with buffer-urea solutions. The (un)folding reactions were monitored by fluorescence by exciting the single Trp at $280 \mathrm{~nm}$ and measuring the emitted light using a $320 \mathrm{~nm}$ cut-off filter. In each case the major folding phase was fitted to an exponential equation and the observed rate constant, $k_{\mathrm{obs}}$, was plotted versus urea concentration and analyzed as described in the next section. The experiments were performed at $25^{\circ} \mathrm{C}$ in $50 \mathrm{mM}$ potassium acetate, $\mathrm{pH}$ 5.4-5.6 or in $50 \mathrm{mM}$ potassium phosphate, $\mathrm{pH}$ 7.0-7.5 with or without sodium sulfate as indicated in the figures. 
Transient aggregation was ruled out by performing refolding experiments at various final concentration of protein $(0.5-4 \mu \mathrm{M})$. The $k_{\mathrm{obs}}$ values thus obtained were independent on protein concentration.

\subsection{Analysis of data}

The dependence of the observed rate constant on urea concentration (chevron plot) was analyzed using the Kaleidagraph and Prism (GraphPad) softwares.

The data were analyzed using the following equations:

Refolding rollover, switch between TS1 and TS2:

$\log k_{\mathrm{obs}}=\log \left(k_{\mathrm{U}} \exp \left(m_{\mathrm{TOT}}\left(1-\beta_{\mathrm{T} 2}\right)[\mathrm{U}] / R T\right)+k_{\mathrm{F}} \exp \left(-\left(\beta_{\mathrm{T} 1} m_{\mathrm{TOT}}\right)[\mathrm{U}] / R T\right) /\left(1+k_{\mathrm{F}} / k_{\mathrm{F} 2} \exp \right.\right.$ $\left.\left.\left(m_{\mathrm{TOT}}\left(\beta_{\mathrm{T} 2}-\beta_{\mathrm{T} 1}\right)[\mathrm{U}] / R T\right)\right)\right)$

Unfolding rollover, switch between $\mathrm{T} 2$ and $\mathrm{T} 3$ :

$\log k_{\mathrm{obs}}=\log \left(k_{\mathrm{F} 2} \exp \left(-\left(m_{\mathrm{TOT}} \beta_{\mathrm{T} 2}\right)[\mathrm{U}] / \mathrm{RT}\right)+k_{\mathrm{U} 2} \exp \left(\left(m_{\mathrm{TOT}}-m_{\mathrm{TOT}} \beta_{\mathrm{T} 2}\right)\right.\right.$

$\left.[\mathrm{U}] / R T) /\left(1+k_{\mathrm{U} 2} / k_{\mathrm{U} 3} \exp \left(\left(m_{\mathrm{TOT}}\left(\beta_{\mathrm{T} 3}-\beta_{\mathrm{T} 2}\right)[\mathrm{U}]\right) / R T\right)\right)\right)$

No rollover, TS2 rate limiting:

$\log k_{\mathrm{obs}}=\log \left(k_{\mathrm{F} 2} \exp \left(-\left(m_{\mathrm{TOT}} \beta_{\mathrm{T} 2}\right)[\mathrm{U}] / R T\right)+k_{\mathrm{U} 2} \exp \left(m_{\mathrm{TOT}}\left(1-\beta_{\mathrm{T} 2}\right)[\mathrm{U}] / R T\right)\right)$

Refolding rollover, switch from TS1 to TS3 (for nNOS PDZ):

$\log k_{\mathrm{obs}}=\log \left(k_{\mathrm{U}} \exp \left(m_{\mathrm{TOT}}\left(1-\beta_{\mathrm{T} 3}\right)[\mathrm{U}] / R T\right)+k_{\mathrm{F}} \exp \left(-\left(\beta_{\mathrm{T} 1} m_{\mathrm{TOT}}\right)[\mathrm{U}] / R T\right) /\left(1+k_{\mathrm{F}} / k_{\mathrm{F} 3} \exp \right.\right.$ $\left.\left.\left(m_{\mathrm{TOT}}\left(\beta_{\mathrm{T} 3}-\beta_{\mathrm{T} 1}\right)[\mathrm{U}] / R T\right)\right)\right)$ 
where $R T$ is $0.59 \mathrm{kcal} \mathrm{mol}^{-1}$ at $25^{\circ} \mathrm{C}$ and [U] is the concentration of urea or $\mathrm{GndHCl}$. $m_{\text {TOT }}$ is the total kinetic $m$-value for the folding reaction and is related to the sum of the slopes of $\log k_{\mathrm{obs}}$ versus [U] in the chevron plot [11].

\section{Results and Discussion}

We included six different PDZ domains in the current study, SAP97 PDZ2, PSD-95 PDZ1, PSD-95 PDZ2, PSD-95 PDZ3, PTP-BL PDZ2 and nNOS PDZ. By changing the buffer conditions in a protein folding experiment ( $\mathrm{pH}$, salt concentration) it is possible to selectively stabilize or destabilize the ground states (the denatured state D and the native state $\mathrm{N}$ ) as well as intermediates and transition states along the folding pathway. Using this approach, we previously found that all tested canonical PDZ domains fold via two transition states separated by a high-energy intermediate [5]. We here extended this study to examine the folding of SAP97 PDZ2 and PSD-95 PDZ2 under stabilizing conditions, i.e., in the presence of 0.4-0.6 M sodium sulfate.

The addition of 0.6 M sodium sulfate to the folding reaction of SAP97 PDZ2 gives a very long and curved refolding arm (Fig. 2A). Conversely, performing the folding experiment under destabilizing conditions ( $50 \mathrm{mM}$ potassium acetate, $\mathrm{pH}$ 5.6) gives a long and curved unfolding arm. Curvatures or "rollovers" indicate that there is a change in rate limiting step for the (un)folding reaction upon changes in denaturant concentration. The presence of two rollovers for the same protein suggests that there are two changes of rate-limiting transition state. We observe the same rollovers for PSD-95 PDZ2 (Fig. 2B). In presence of 0.4 M sodium sulfate there is as rollover in 
the refolding arm, and in $50 \mathrm{mM}$ potassium acetate, $\mathrm{pH} 5.5$ there is a rollover in the unfolding arm as reported previously [5] (Fig. 2B).

The position of a transition state along the reaction coordinate for protein folding is often described by the $\beta_{\mathrm{T}}$-value. This value, which can be between 0 and 1 , correlates well with the degree of burial of solvent accessible surface area during the folding reaction [12]. Because of this correlation, the $\beta_{\mathrm{T}}$ value is a measure of the compactness of the protein, which is used as the reaction coordinate for protein folding. The denatured state has a $\beta_{\mathrm{T}}$-value of 0 and the native state a value of 1 (see Fig. 4). The $\beta_{\mathrm{T}}$-value for a transition state is calculated from the slopes of the refolding and unfolding limbs of the chevron plot, as described previously [13].

Chi et al. [5] found that the $\beta_{\mathrm{T}}$-values could be shared for all their tested PDZ domains with unfolding arm rollovers. These similar $\beta_{\mathrm{T}}$-values suggested that the respective reaction pathway had transition state structures of similar compactness and that these PDZ domains shared a conserved folding mechanism with two transition states separated by a high-energy intermediate. Moreover, the nNOS PDZ domain with a refolding arm rollover could also be satisfactorily fitted to the same parameters suggesting that it is possible that the shift between the two similar transition states can cause a rollover in the unfolding arm for certain PDZ domains and in the refolding arm for other ones. While this is possible, a more complex, yet more likely scenario to explain the refolding rollover for nNOS PDZ, in light of our present data on SAP97 PDZ2 and PSD-95 PDZ2, is a sequential pathway that has two high-energy intermediates and three transition states. The change of rate-limiting step from the 
second to the first transition state gives the refolding arm rollover and the change in rate-limiting step from the second to the third creates the unfolding arm rollover.

We fitted our data on SAP97 PDZ2 and PSD-95 PDZ2, respectively, with shared $\beta_{\mathrm{T}^{-}}$ values to such a sequential pathway (Fig 2). In the work by Chi et al. [5] the $\beta_{\mathrm{T}}$-values were $0.66 \pm 0.01(\mathrm{TS} 2)$ and $0.93 \pm 0.04$ (TS3) for the whole PDZ dataset, which is similar to the values obtained for SAP97 PDZ2 and PSD-95 PDZ2. We then re-fitted the data from Chi et al. [5], together with our new data according to the three-TS model (Fig. 3). The folding kinetics of all six PDZ domains fit nicely to this scheme with $\beta_{\mathrm{T}}$-values as reported in the legend to Fig. 3.

By adjusting the experimental conditions, all three TSs can be revealed in the folding reactions of SAP97 PDZ2 and PSD-95 PDZ2. For nNOS PDZ, TS2 is not visible but the switch in rate-limiting step is from TS1 directly to TS3. For PTP-BL PDZ2, the refolding arm is linear even in presence of $0.4 \mathrm{M}$ sodium sulfate [7], that is, only the switch from TS2 to TS3 is visible. These results are all in line with detailed structural analyses of TS2 and TS3 [14], which show that late events (TS3) are conserved for the folding reaction within the PDZ family. TS2 on the other hand is more different and it is likely that the structures of very early intermediates and transition states diverge even more in terms of structure. For example, the species corresponding to a $\beta_{\mathrm{T}}$-value of 0.20 in the folding reaction of PTP-BL PDZ1 is of lower energy and never becomes rate limiting, in contrast to those of PSD-95 PDZ2 and SAP97 PDZ2.

In summary, despite a low degree of sequence identity, the folding reactions of all PDZ domains considered are consistent with a common mechanism. Within this 
frame, it is possible to conclude that protein topology determines the mechanism of folding of a given protein, whereas its sequence composition tunes the relative stabilities of the different folding transition states are intervening intermediates. Fig. 4 summarizes the unified model for the folding of PDZ domains.

\author{
Abbreviations \\ nNOS, neuronal nitric oxide synthase; PDZ, PSD-95/Discs large/ZO-1; PSD-95, \\ Postsynaptic density protein-95
}

\title{
Acknowledgments
}

This work was supported by the Swedish Research Council (grant 2009-5659, to P.J.) and by the Italian Ministero dell'Istruzione dell'Universita`e della Ricerca (Progetto di Interesse 'Invecchiamento' (to S.G.)

\section{References}

[1] S. Gianni, Y. Ivarsson, P. Jemth, C. Travaglini-Allocatelli, M. Brunori, Identification and characterization of protein folding intermediates, Biophysical Chemistry 128 (2007) 105-113.

[2] B. G. Wensley, S. Batey, F. A. Bone, Z. M. Chan, N. R. Tumelty, A. Steward, L. G. Kwa, A. Borgia, J. Clarke, Experimental evidence for a frustrated energy landscape in a three-helix-bundle protein family, Nature 463 (2010) 685-688.

[3] T. Cellmer, E. R. Henry, J. Hofrichter, W. A. Eaton, Measuring internal friction of an ultrafast-folding protein, Proc. Natl. Acad. Sci. U S A 105 (2008) 18320-18325.

[4] L. L. Chavez, J. N. Onuchic, C. Clementi, Quantifying the roughness on the free energy landscape: entropic bottlenecks and protein folding rates, J. Am. Chem. Soc. 126 (2004) 8426-8432.

[5] C. N. Chi, S. Gianni, N. Calosci, C. Travaglini-Allocatelli, Å. Engström, P. Jemth, A conserved folding mechanism for PDZ domains, FEBS Lett. 581 (2007) 1109-1113.

[6] S. Gianni, N. Calosci, J. Aelen, G. W. Vuister, M. Brunori, C. TravagliniAllocatelli, Kinetic folding mechanism of PDZ2 from PTP-BL, 18 (2005) 389-395. 
[7] S. Gianni, C. D. Geierhaas, N. Calosci, P. Jemth, G. W. Vuister, C. Travaglini-Allocatelli, M. Vendruscolo, M. Brunori, A PDZ domain recapitulates the unifying mechanism for protein folding, Proc. Natl. Acad. Sci. USA 104 (2007) 128-133.

[8] P. Jemth, S. Gianni, PDZ domains: folding and binding, Biochemistry 46 (2007) 8701-8708.

[9] E. Kim, M. Sheng, PDZ domain proteins of synapses, Nat. Rev. Neurosci. 5 (2004) 771-781.

[10] S. R. Haq, M. C. Jürgens, C. N. Chi, C. S. Koh, L. Elfström, M. Selmer, S. Gianni, P. Jemth, The plastic energy landscape of protein folding: a triangular folding mechanism with an equilibrium intermediate for a small protein domain, J. Biol. Chem. 285 (2010) 18051-18059.

[11] S. E. Jackson, A. R. Fersht, Folding of chymotrypsin inhibitor 2. 1. Evidence for a two-state transition, Biochemistry 30 (1991) 10428-10435.

[12] J. K. Myers, C. N. Pace, J. M. Scholtz, Denaturant m values and heat capacity changes: relation to changes in accessible surface areas of protein unfolding, Protein Sci. 4 (1995) 2138-2148.

[13] A. Fersht (1999) Structure and Mechanism in Protein Science, W.H. Freeman and Co., New York.

[14] N. Calosci, C. N. Chi, B. Richter, C. Camilloni, Å. Engström, L. Eklund, C. Travaglini-Allocatelli, S. Gianni, M. Vendruscolo, P. Jemth, A comparison of the successive transition states for folding reveals alternative early folding pathways, Proc. Natl. Acad. Sci. U S A 105 (2008) 19241-19246. 


\section{Figures}

Fig. 1. Crystal structure of SAP97 PDZ2 (pdb code: $2 x 7 z$ ) [10], showing the Trp used for monitoring the folding reaction.

Fig. 2. Chevron plots of SAP97 PDZ2 $(A)$ and PSD-95 PDZ2 $(B)$ under different conditions. The data were fitted to the equations described in section 2.3. SAP97 PDZ2: $\beta_{\mathrm{T} 1}=0.18 \pm 0.07, \beta_{\mathrm{T} 2}=0.70 \pm 0.01, \beta_{\mathrm{T} 3}=0.96 \pm 0.11$. PSD-95 PDZ2: $\beta_{\mathrm{T} 1}=$ $0.05 \pm 0.13, \beta_{\mathrm{T} 2}=0.65 \pm 0.02, \beta_{\mathrm{T} 3}=1.0 \pm 0.10$

Fig. 3. Chevron plots for SAP97 PDZ2, PSD-95 PDZ1, PSD-95 PDZ2, PSD-95 PDZ3, nNOS PDZ and PTP-BL PDZ2, obtained under different conditions. Data from Fig. 2 and Chi et al. [5] were fitted to the model with three transition states separated by two high-energy intermediates. The $\beta_{\mathrm{T}}$ values were shared among all datasets in the curve fitting: $\beta_{\mathrm{T} 1}=0.17 \pm 0.04, \beta_{\mathrm{T} 2}=0.65 \pm 0.01, \beta_{\mathrm{T} 3}=0.86 \pm 0.01$ (curve fitting errors).

Fig. 4. Energy diagram showing the unified model for folding of PDZ domains, with three transitions states (TS1, TS2 and TS3) separated by two high-energy intermediates. The relative energy levels in the figure are at low urea concentration and under stabilizing conditions such that TS1 is rate limiting for folding. D, denatured state; $\mathrm{N}$, native state. 


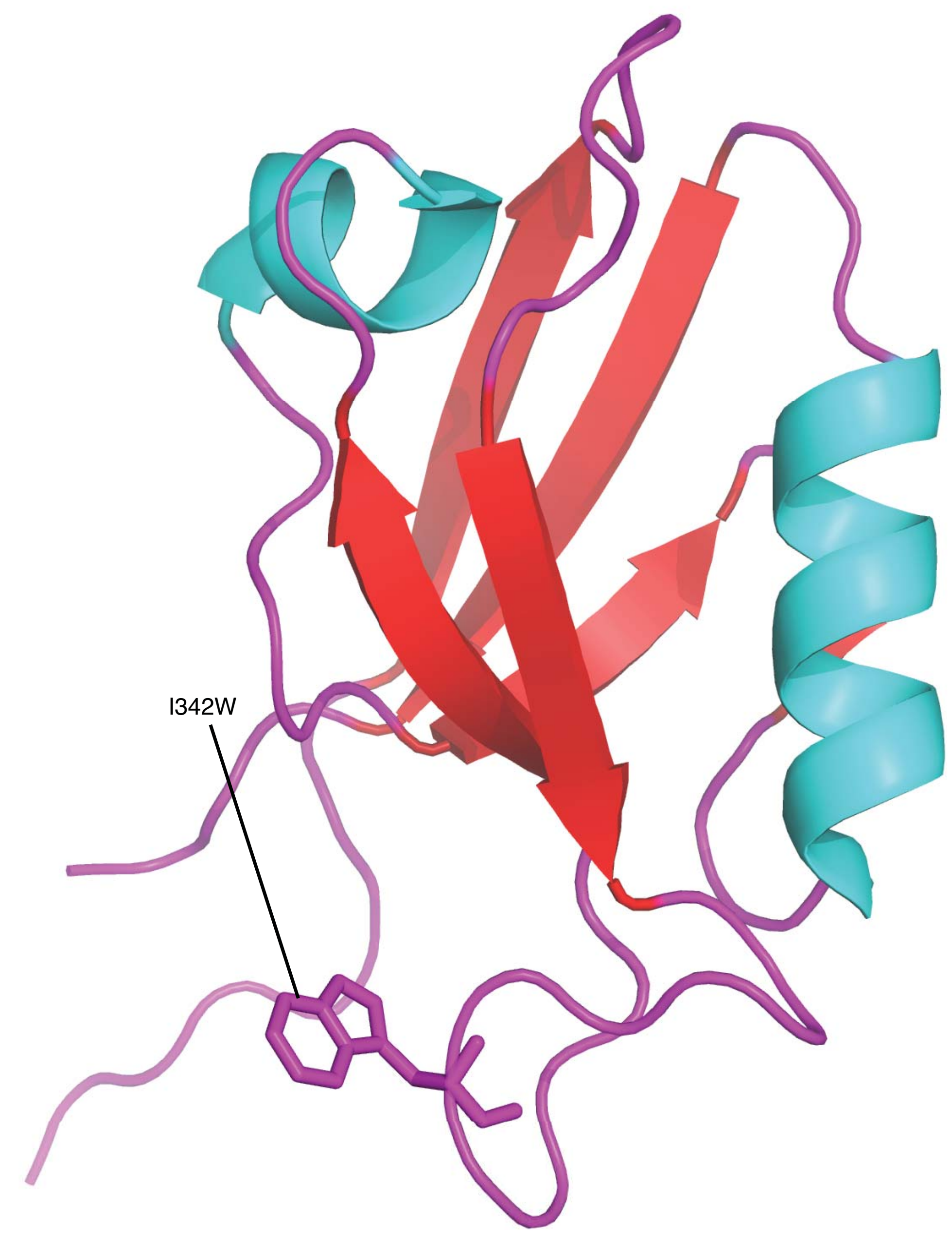




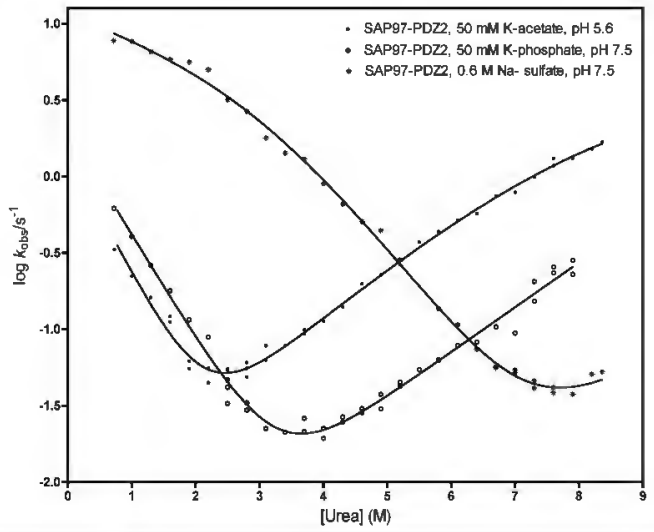




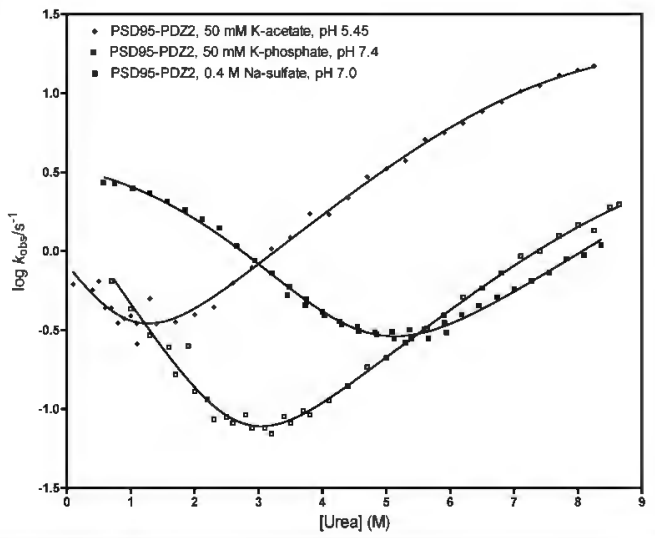




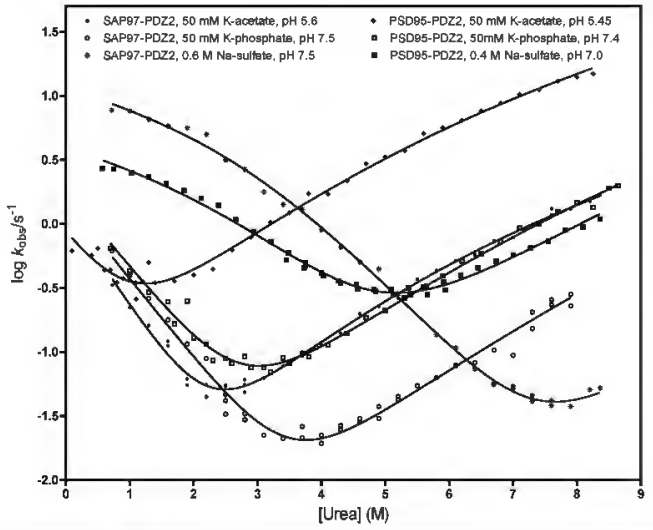




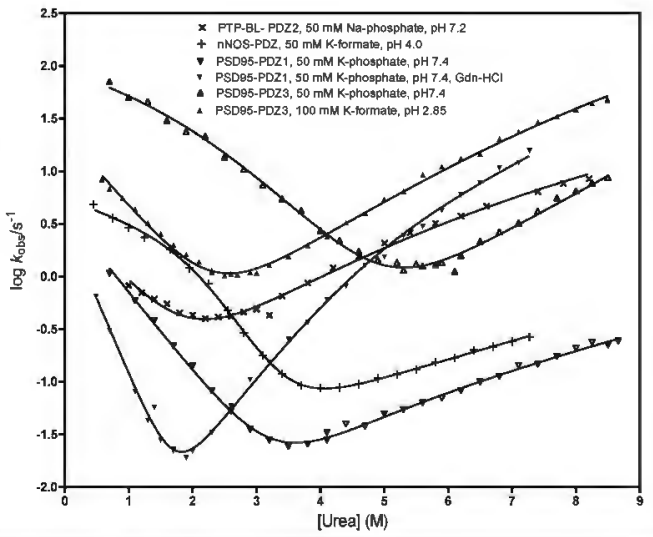




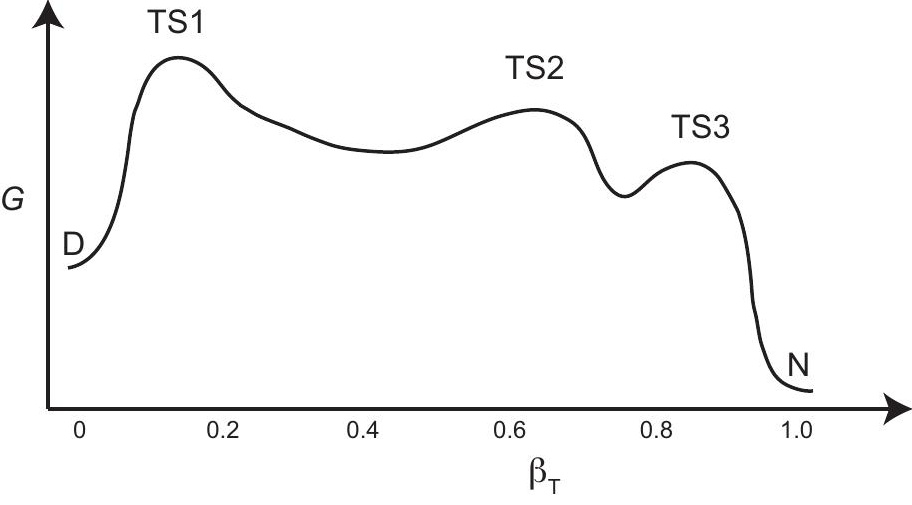




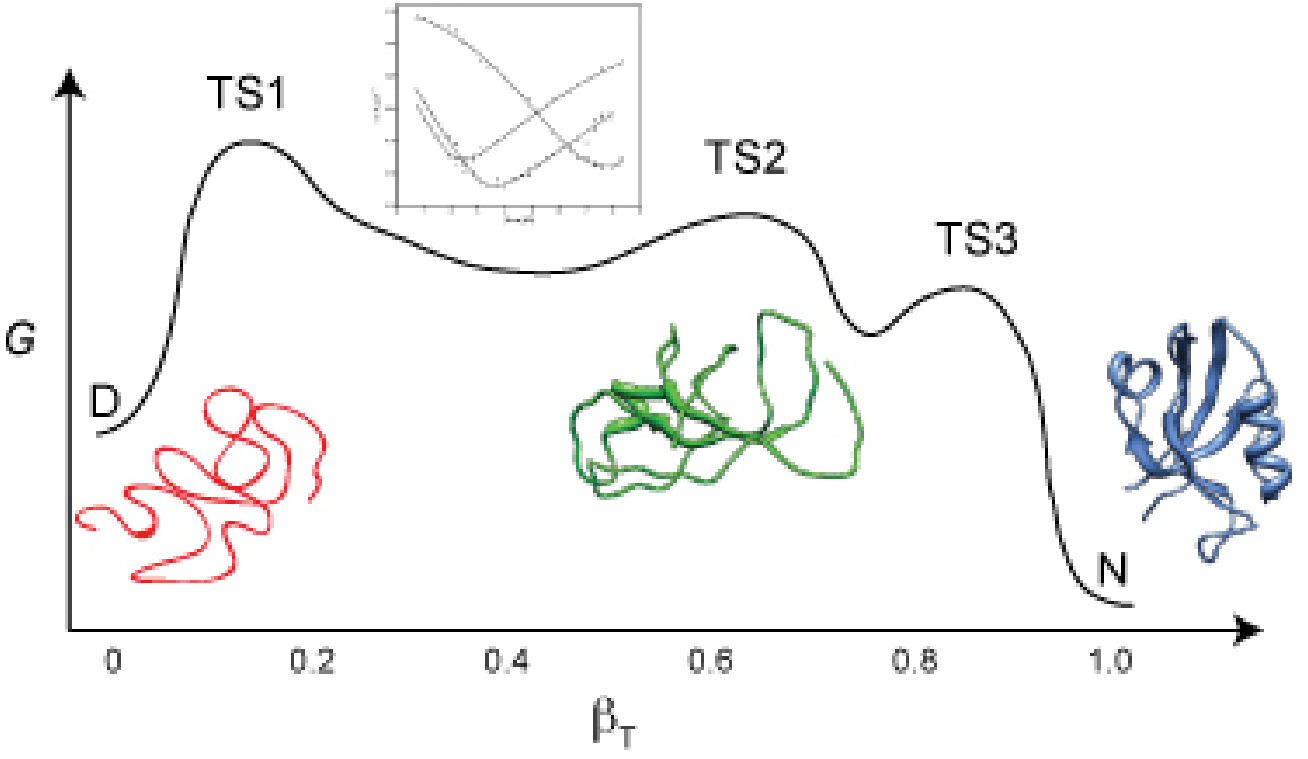

